\title{
A simultaneous multiresidue analysis for 203 pesticides in soybean using florisil solid-phase extraction and gas chromatography- tandem mass spectrometry
}

\author{
Yongho Shin ${ }^{1} \cdot J^{\prime}$ gnghwa Lee ${ }^{1} \cdot$ Jeong-Han Kim $^{1}[$
}

Received: 26 June 2018/ Accepted: 19 July 2018/Published online: 2 August 2018

(C) The Korean Society for Applied Biological Chemistry 2018

\begin{abstract}
A multiresidue analysis method for the simultaneous determination of 203 pesticides in soybean was developed using solid-phase extraction (SPE) and gas chromatography-tandem mass spectrometry (GC-MS/ MS). Scheduled multiple reaction monitoring by GC-MS/ MS was optimized in electron ionization mode. The target pesticides satisfied the method limit of quantitation below $0.01 \mathrm{mg} / \mathrm{kg}$, and an excellent instrumental repeatability was obtained. The calibration curve correlation coefficients $\left(r^{2}\right)$ for $201(99.0 \%)$ pesticides were $\geq 0.990$. The Multiclass Pesticide Multiresidue Method (No. 2) of the Korea Food Code was scaled down and applied for efficient sample treatment. Soybean sample $(10 \mathrm{~g})$ was extracted with $20 \mathrm{~mL}$ acetonitrile. The sample was filtered, partitioned with sodium chloride, and centrifuged. The supernatant $(8 \mathrm{~mL})$ was subjected to cleanup with a florisil SPE cartridge $(500 \mathrm{mg}$ ), and the final extract was subjected to GC-MS/MS analysis. To remove fat, further liquid-liquid partitioning with $n$-hexane/acetonitrile was conducted before the SPE cleanup, and this procedure was compared to the non-partitioning procedure. The percentage of pesticides satisfying the recovery range of $70-120 \%$ with a relative standard deviation $\leq 20 \%$ in the non-partitioning and partitioning procedures were $87.2 \%$ and $78.8 \%$ at $0.01 \mathrm{mg} / \mathrm{kg}$ and $88.2 \%$ and $76.8 \%$ at $0.05 \mathrm{mg} / \mathrm{kg}$,
\end{abstract}

Electronic supplementary material The online version of this article (https://doi.org/10.1007/s13765-018-0388-y) contains supplementary material, which is available to authorized users.

Jeong-Han Kim

kjh2404@snu.ac.kr

1 Department of Agricultural Biotechnology and Research Institute of Agriculture and Life Sciences, Seoul National University, Seoul 08826, Republic of Korea respectively. The average matrix effect value (\%) was $5.5 \%$. This miniaturized analytical method can be successfully applied for rapid and sensitive monitoring of multiresidues in soybean and related agricultural products.

Keywords GC-MS/MS · Multiresidue · Pesticide · Positive list system - Solid-phase extraction - Soybean

\section{Introduction}

Pesticide residues in agricultural products are regulated by governmental authorities to secure the health of populations. The current food safety system for pesticide residues in the Republic of Korea is based on the negative list system (NLS), in which the pesticide residue according to maximum residue levels (MRLs) list is regulated [1]; however, the positive list system (PLS) will be enforced from 2019 onward instead of the NLS. In the PLS, the levels of pesticide residue should be below $0.01 \mathrm{mg} / \mathrm{kg}$ when an MRL has not been established in corresponding crops and food [2]. The PLS has been successfully implemented for many years in various countries including the USA, Australia, Canada, Hong Kong, Japan, Taiwan, and the European Union (EU). In Korea, the PLS has been implemented for tropical and subtropical fruits, nuts, and seeds since December 31, 2016, and will be applied to all agricultural products starting on January 1, 2019 [1].

The introduction of the PLS requires high-throughput analytical techniques with reliable accuracy to determine trace concentrations of hundreds of pesticides in various food matrices, with a short analysis time. Gas chromatography-tandem mass spectrometry (GC-MS/MS) has been widely used as an analytical tool to simultaneously detect 
and quantify multiresidue pesticides with high efficiency [3-5]. The multiple reaction monitoring (MRM) mode of GC-MS/MS is able to distinguish target analytes from interferences with high sensitivity and selectivity. The Multiclass Pesticide Multiresidue Method (No. 2) of the Korea Food Code is a powerful sample treatment procedure enabling the determination of pesticide multiresidues in various agricultural products [6]. In this method, the florisil solid-phase extraction (SPE) is applied, using polar magnesium silicate for adsorption chromatography in a cleanup step. Florisil has strong purification characteristics for pesticides from fatty samples due to its ability to preferentially retain some lipids [7]. Polar matrices such as triglycerides and phytosterols are also easily removed from the sample extract by associating with the surface of florisil [8].

Soybean is one of the most important agricultural products worldwide due to its higher protein content than other vegetables, and it is cultivated on an approximately $6 \%$ of the world's arable lands [9]. It has a high fat content (20\% of total weight) [10] and is thus used as an edible oil. There are 112 and 481 MRLs for soybean established in Korea [11] and the EU [12], respectively; however, few studies have analyzed pesticide multiresidues in soybean.

In this study, we selected 203 GC-amenable pesticides from the soybean MRL lists of Korea and the EU and developed a simultaneous analytical method using GCMS/MS. The scheduled MRM mode of GC-MS/MS was used as an effective throughput of target pesticides. The official Multiclass Pesticide Multiresidue Method (No. 2) of the Korea Food Code was scaled down and validated with 203 pesticides. To remove fat, a liquid-liquid partitioning method using $n$-hexane/acetonitrile was also investigated for comparison with the non-partitioning method.

\section{Materials and methods}

\section{Chemicals and reagents}

Each of pesticide standard (analytical grade) and stock solution was purchased from ChemService (West Chester, PA, USA), Wako Pure Chemical Industries (Osaka, Japan), Dr. Ehrenstorfer (Augsburg, Germany), Sigma-Aldrich (St. Louis, MO, USA), AccuStandard (New Haven, CT, USA), and ULTRA Scientific (North Kingstown, RI, USA) and gratefully obtained from the Ministry of Food and Drug Safety (Republic of Korea). High-performance liquid chromatography (HPLC)-grade acetonitrile, acetone, and $n$-hexane were sourced from Fisher Scientific (Seoul, Republic of Korea). Sodium chloride $(\mathrm{NaCl}, 99.0 \%)$ was obtained from Samchun (Gyeonggi-do, Republic of
Korea). Diethylene glycol ( $\geq 99.0 \%$ ) was purchased from Sigma-Aldrich. A Strata-FL-PR florisil cartridge $(500 \mathrm{mg} /$ $6 \mathrm{~mL}$ ) was obtained from Phenomenex (Torrance, CA, USA).

\section{Instrumental conditions (GC-MS/MS)}

Pesticide multiresidues were analyzed with the GCMSTQ8040 triple quadrupole mass spectrometer (Shimadzu, Kyoto, Japan). A Topaz glass liner $(3.5 \mathrm{~mm})$ with wool (Restek, Bellefonte, PA) was installed in the inlet, and the capillary column was an Rxi-5Sil MS $(30 \mathrm{~m} \times 0.25 \mathrm{~mm}$ i.d., $0.25 \mu \mathrm{m} d_{f}$, Restek). The inlet temperature was $280{ }^{\circ} \mathrm{C}$, and the injection volume was $2 \mu \mathrm{L}$. The splitless (75 s) mode was used during sample injection. The oven temperature program was initialized at $70{ }^{\circ} \mathrm{C}$ (held for $2 \mathrm{~min}$ ), ramped to $160{ }^{\circ} \mathrm{C}$ at $15^{\circ} \mathrm{C} / \mathrm{min}$, increased to $260{ }^{\circ} \mathrm{C}$ at $5{ }^{\circ} \mathrm{C} / \mathrm{min}$, and finally ramped to $300{ }^{\circ} \mathrm{C}$ at $15^{\circ} \mathrm{C} /$ min (held for $8 \mathrm{~min}$ ). The total program time was $38.7 \mathrm{~min}$. Helium ( $\geq 99.999 \%$ ) was used as a carrier gas, and the flow rate (constant) was $1.0 \mathrm{~mL} / \mathrm{min}$.

For MS/MS, the electron ionization (EI) mode was selected for ionization and the electron voltage was $70 \mathrm{eV}$. The ion source and transfer line temperatures were 230 and $280{ }^{\circ} \mathrm{C}$, respectively. The collision inductive dissociation (CID) was assisted with argon $(\geq 99.999 \%)$ gas. The detector voltage was $1.4 \mathrm{kV}$ (constant). Data processing was conducted using LabSolutions (GCMS solution, version 4.30).

\section{MRM profile optimization}

Each pesticide standard solution was injected into GC-MS/ MS, and a full-scan spectrum of each target analyte was obtained (m/z range; $50-500)$. From the spectrum, one or two fragment(s) or molecular ion(s) were selected as a precursor ion(s) considering their specificity. Each precursor ion was subjected to a product scan using CID with various collision energies, and two optimum product ions were selected as quantifier and qualifier ions, respectively.

\section{Method limit of quantitation, instrumental repeatability, and linearity of calibration}

The method limit of quantitation (MLOQ) was calculated from the instrumental limit of quantitation (ILOQ), injection volume, and sample equivalent in the final extract using Eq. (1):

$$
\begin{aligned}
\operatorname{MLOQ}\left(\mathrm{mg} \mathrm{kg}^{-1}\right)= & \frac{\operatorname{ILOQ}(\mathrm{ng})}{\text { injection volume }(\mu \mathrm{L})} \\
& \times \frac{1}{\text { sample equivalent }\left(\mathrm{g} \mathrm{mL}^{-1}\right)} .
\end{aligned}
$$


Matrix-matched standards in the soybean samples were injected into GC-MS/MS, and the lowest amount that satisfied a signal-to-noise ratio $(\mathrm{S} / \mathrm{N}) \geq 10$ for each pesticide was selected as the ILOQ. The instrumental repeatability for each pesticide was verified with the relative standard deviation (RSD) of the peak area by injecting and analyzing a matrix-matched standard seven times. The linearity of calibration expressed as correlation coefficient $\left(r^{2}\right)$ was investigated using matrix-matched standards $(0.002-0.2 \mathrm{mg} / \mathrm{L})$. A weighting regression factor $(1 / x)$ was used to minimize calculation errors at low concentrations.

\section{Recovery test without a defatting step using $n$-hexane/acetonitrile partitioning}

Sample treatment was conducted using Multiclass Pesticide Multiresidue Method (No. 2) with a modification of sample weight (i.e., a $1 / 5$ reduction). Ten grams of macerate was spiked at $0.01 \mathrm{mg} / \mathrm{kg}(n=3)$ and $0.05 \mathrm{mg} / \mathrm{kg}$ ( $n=3$ ) of 203 pesticides, and $6 \mathrm{~mL}$ water was added to let the entire sample soak the water before extraction with $20 \mathrm{~mL}$ acetonitrile. The tube was shaken for $2 \mathrm{~min}$ at $1200 \mathrm{rpm}$ using the Geno/Grinder ${ }^{\circledR}$ (1600 MiniG SPEX Sample Prep, Metuchen, NJ, USA), and then, the sample was subjected to suction filtration under vacuum. The extract was treated with $3 \mathrm{~g} \mathrm{NaCl}$, shaken, and centrifuged for $5 \mathrm{~min}$ at $3500 \mathrm{rpm}$ using the Combi 408 centrifuge (Hanil Science Industrial Co., Ltd., Republic of Korea). The supernatant $(8 \mathrm{~mL})$ was combined with $0.2 \mathrm{~mL}$ of $2 \%$ diethylene glycol (keeper solution) in acetone, evaporated with a rotary evaporator $\left(40{ }^{\circ} \mathrm{C}\right)$, and reconstructed in $4 \mathrm{~mL}$ of $20 \%$ acetone in $n$-hexane (v/v).

For the SPE cleanup step, a florisil cartridge (500 mg) was conditioned with $5 \mathrm{~mL} n$-hexane and $5 \mathrm{~mL}$ of $20 \%$ acetone in $n$-hexane. Reconstructed extract $(4 \mathrm{~mL})$ was loaded and the cartridge was eluted again with $5 \mathrm{~mL}$ of $20 \%$ acetone in $n$-hexane. The eluted extracts were evaporated to dryness under a stream of nitrogen gas. The sample was dissolved in $2 \mathrm{~mL}$ acetonitrile, and $0.8 \mathrm{~mL}$ of the extract was matrix-matched with $0.2 \mathrm{~mL}$ of acetonitrile. Finally, the aliquot $(2 \mu \mathrm{L})$ was injected into GC-MS/ MS for analysis. The sample was equivalent to $1.6 \mathrm{~g}$ per $1 \mathrm{~mL}$ in the final extract.

\section{Recovery test with a defatting step using $\boldsymbol{n}$-hexane/ acetonitrile partitioning}

Ten grams of soybean macerate containing $0.01 \mathrm{mg} / \mathrm{kg}$ $(n=3)$ and $0.05 \mathrm{mg} / \mathrm{kg}(n=3)$ of 203 pesticides was subjected to extraction, filtration, $\mathrm{NaCl}$ partitioning, and evaporation as described above. Then, the extract was dissolved in $30 \mathrm{~mL} n$-hexane saturated with acetonitrile and partitioned with $30 \mathrm{~mL}$ acetonitrile saturated with $n$ hexane (twice). The lower layers from each partition were collected, treated with $0.2 \mathrm{~mL}$ of $2 \%$ diethylene glycol in acetone, and evaporated with a rotary evaporator $\left(40{ }^{\circ} \mathrm{C}\right)$ for the next cleanup step and analysis as described above. Recovery of this partitioning step with $n$-hexane/acetonitrile in this method was compared to the method without a partitioning (non-partitioning) procedure.

\section{Matrix effect}

The matrix effect was verified to compare the slope of calibration from a matrix-matched standard and from a solvent-only standard. Matrix effect values (\%) for each pesticide were calculated using Eq. (2):

$$
\begin{aligned}
& \text { Matrix effect }(\%) \\
& =\left(\frac{\text { Slope of matrixmatched standard calibration }}{\text { Slope of solventonly standard calibration }}-1\right) \times 100 .
\end{aligned}
$$

\section{Results and discussion}

\section{MRM optimization and MLOQ}

Using each pesticide standard, MRM profiles were successfully established and also verified under GC conditions. The detailed MRM profiles and retention time $\left(t_{\mathrm{R}}\right)$ for 203 pesticides are given in Table S1.

The ILOQ was determined by the S/N method [13]. Matrix-matched standard mixtures at $0.004,0.01,0.02$, $0.04,0.1,0.2$ and $0.4 \mathrm{ng}$ (equivalent to $0.002,0.005,0.01$, $0.02,0.05,0.1$ and $0.2 \mathrm{mg} / \mathrm{L}$ of the matrix-matched standard solutions) were injected into the GC-MS/MS, respectively, and the lowest amount satisfying an $\mathrm{S} / \mathrm{N} \geq 10$ on the chromatogram was selected as the ILOQ. The MLOQ was derived from the ILOQ, injection volume $(2 \mu \mathrm{L})$, and sample equivalent $(1.6 \mathrm{~g} / \mathrm{mL})$ (see Eq. 1). As a result, all of the pesticides had MLOQ $<0.01 \mathrm{mg} / \mathrm{kg}(193$ compounds for $<0.002 \mathrm{mg} / \mathrm{kg}, 7$ for $0.003 \mathrm{mg} / \mathrm{kg}$, and 3 for $0.006 \mathrm{mg} / \mathrm{kg}$ ) (Table S2). This indicates that these pesticides are applicable for the PLS, in which the residue level should be under $0.01 \mathrm{mg} / \mathrm{kg}$ for a pesticide without the Korean MRL lists [1]. Thus, this analytical method can determine pesticide multiresidues in soybean and related crop with sufficient sensitivity.

\section{Instrumental repeatability}

Instrumental repeatability is a parameter that ensures the integrity of instrumental performance for target compounds $[14,15]$. A repeatability test was conducted by consecutively injecting matrix-matched standard solutions at 0.01 
and $0.05 \mathrm{mg} / \mathrm{L}$ ( $n=7$, respectively), and the area RSD was verified for each pesticide. The average RSDs of the 203 target analytes were $2.4 \%$ at $0.01 \mathrm{mg} / \mathrm{L}$ and $1.4 \%$ at $0.05 \mathrm{mg} / \mathrm{L}$ (Table $\mathrm{S} 2$ ), with a better repeatability at higher concentration. Among the pesticides, 200 (98.5\%) compounds at $0.01 \mathrm{mg} / \mathrm{L}$ and $203(100.0 \%)$ at $0.05 \mathrm{mg} / \mathrm{L}$ were below than $10 \%$ (Table 1). This indicates that GC-MS/MS had excellent performance for the analysis of most pesticides. The RSDs for three $(1.5 \%)$ of the pesticides at $0.01 \mathrm{mg} / \mathrm{L}$ fell within $10.0-12.4 \%$. These analytes still had an acceptable repeatability, considering that the RSD criterion for recovery was $\leq 20 \%$ according to the SANTE guidance document [16].

\section{Linearity of calibration}

The linearity of matrix-matched calibration (weighting regression factor, $1 / x$ ) was determined. Among the 203 target analytes, $201(99.0 \%)$ pesticides had a $r^{2} \geq 0.990$ (Fig. 1). Only the $r^{2}$ values of methoxychlor and $p, p^{\prime}$-DDT were lower at 0.980-0.990 and $<0.980$, respectively. Therefore, precise quantitative analysis was possible for most of the pesticides. The $r^{2}$ values for the 203 pesticides are presented in Table S2.

\section{Recovery without $\boldsymbol{n}$-hexane/acetonitrile partitioning (non-partitioning)}

For efficient sample treatment, the sample size and extraction solvent volume used in the Multiclass Pesticide Multiresidue Method (No. 2) were reduced from 50 to $10 \mathrm{~g}$ and 100 to $20 \mathrm{~mL}$ acetonitrile, respectively. During the evaporation at $40{ }^{\circ} \mathrm{C}$, diethylene glycol in acetone, a keeper solution, was added to each sample to prevent some volatile pesticides from evaporating with the solvent [17]. To maintain the optimal and rugged performance of the florisil SPE, the sorbent was prevented from drying out during the conditioning, sample loading, and elution steps, as described by Mutavdžić et al. [18].

The recovery test was conducted at 0.01 and $0.05 \mathrm{mg} /$ $\mathrm{kg}$. It was found that $177(87.2 \%)$ and $179(88.2 \%)$ of all

Table 1 Distribution of the instrumental repeatability expressed as the area relative standard deviation (RSD) $(n=7)$ at 0.01 and $0.05 \mathrm{mg} / \mathrm{L}$

\begin{tabular}{lcc}
\hline $\begin{array}{l}\text { Area RSD } \\
n=7\end{array}$ & $0.01 \mathrm{mg} / \mathrm{L}$ & $0.05 \mathrm{mg} / \mathrm{L}$ \\
No. of analytes $(\%)$ & No. of analytes $(\%)$ \\
\hline $0-10 \%$ & $200(98.5)$ & $203(100.0)$ \\
$10-20 \%$ & $3(1.5)$ & $0(0.0)$ \\
Sum & $203(100)$ & $203(100)$ \\
\hline
\end{tabular}

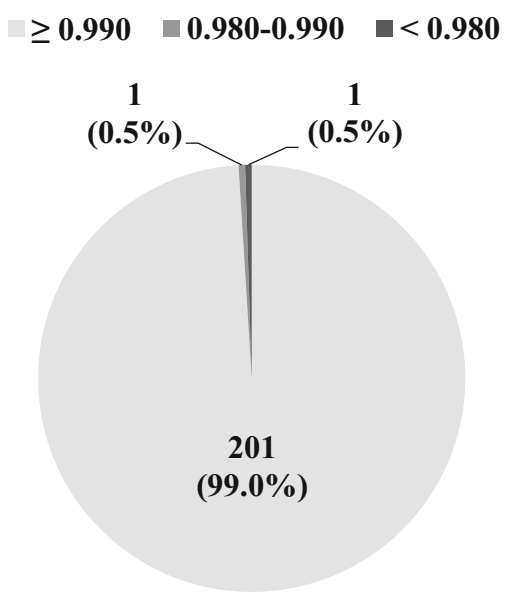

Fig. 1 Pie chart showing the linearity of calibration for 203 pesticides expressed as the correlation coefficient $\left(r^{2}\right)$ (light gray, $\geq 0.990$; gray, 0.980-0.990; dark gray, $<0.980$ )

203 pesticides satisfied the acceptable recovery criteria [16] of 70-120\% with an RSD $\leq 20 \%$ (Table 2) at 0.01 and $0.05 \mathrm{mg} / \mathrm{kg}$, respectively. The number of pesticides satisfying the criteria at $0.05 \mathrm{mg} / \mathrm{kg}$ was slightly higher than at $0.01 \mathrm{mg} / \mathrm{kg}$. These compounds were detectable with a high reliability and precision. The number of pesticides and its percentage satisfying the criteria at $0.01 \mathrm{mg} / \mathrm{kg}$ were higher than those in modified QuEChERS (quick, easy, cheap, effective, rugged, and safe) methods with fatty matrices such as soy-based nutraceutical products and avocado $[19,20]$. The number of pesticides outside the recovery criteria $(30-70 \%)$ but that still within an RSD $\leq 20 \%$ were $26(12.8 \%)$ and $24(11.8 \%)$ at 0.01 and $0.05 \mathrm{mg} / \mathrm{kg}$, respectively. The recovery of these compounds was consistent [16] and therefore acceptable for screening purposes. The detailed recovery rates for 203 pesticides are given in Table S3.

\section{Recovery with $\boldsymbol{n}$-hexane/acetonitrile partitioning}

Because soybean has a high fat content (20\% of total weight) [10], a defatting procedure could be used to

Table 2 Distribution of the recovery ranges for 203 pesticides classified with $30-70 \%(\mathrm{RSD} \leq 20 \%)$ and $70-120 \%(\mathrm{RSD} \leq 20 \%)$ at 0.01 and $0.05 \mathrm{mg} / \mathrm{kg}$

\begin{tabular}{lll}
\hline Recovery range & $\begin{array}{l}0.01 \mathrm{mg} / \mathrm{kg} \\
\text { No. of analytes }(\%)\end{array}$ & $\begin{array}{l}0.05 \mathrm{mg} / \mathrm{kg} \\
\text { No. of analytes }(\%)\end{array}$ \\
\hline $\begin{array}{l}30-70 \% \\
(\mathrm{RSD} \leq 20 \%)\end{array}$ & $26(12.8 \%)$ & $24(11.8 \%)$ \\
$70-120 \%$ & $177(87.2 \%)$ & $179(88.2 \%)$ \\
(RSD $\leq 20 \%)$ & $203(100 \%)$ & $203(100 \%)$ \\
Sum & & \\
\hline
\end{tabular}




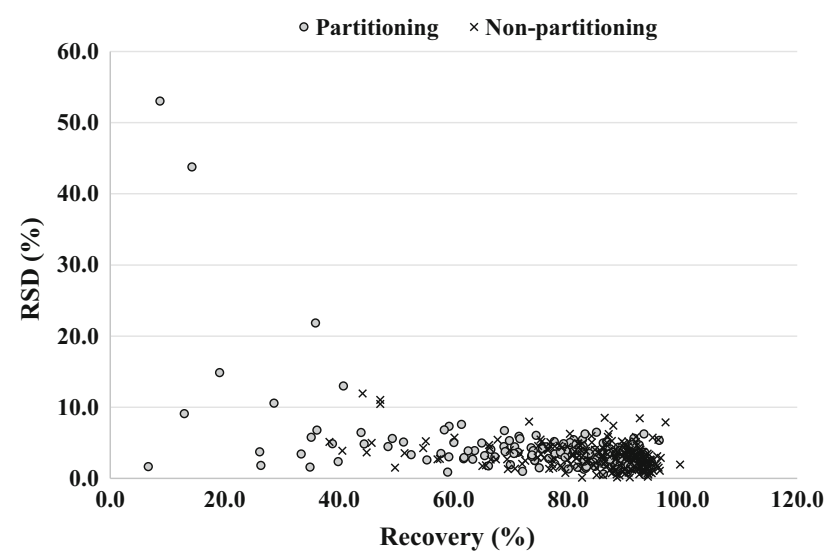

Fig. 2 Scatter plot between recovery and relative standard deviation (RSD) of 203 pesticides for partitioning and non-partitioning procedures $(0.05 \mathrm{mg} / \mathrm{kg})$

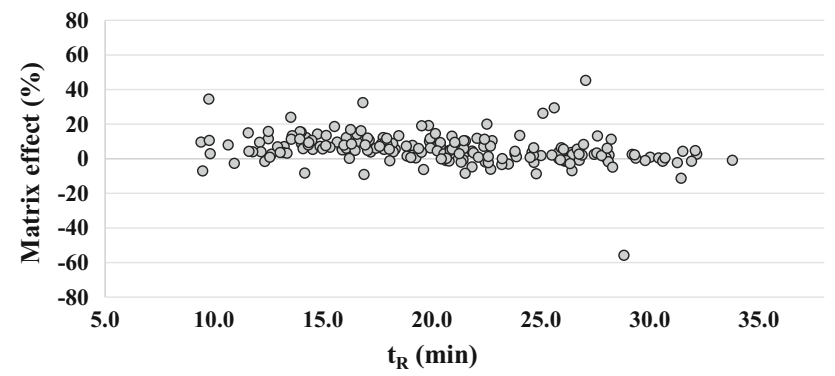

Fig. 3 Scatter plot between the retention time $\left(t_{\mathrm{R}}\right)$ and matrix effect of 203 pesticides

remove fat according to the guidance of the Korea Food Code [6]. Therefore, a recovery test at 0.01 and $0.05 \mathrm{mg} / \mathrm{kg}$ was conducted using a defatting procedure with $n$-hexane/ acetonitrile partitioning. As a result, $160(78.8 \%)$ and 156 (76.8\%) of the 203 pesticides met an acceptable recovery criteria of $70-120 \%$ with an RSD $\leq 20 \%$ [16] at 0.01 and
$0.05 \mathrm{mg} / \mathrm{kg}$ (Table S3), respectively. The number of pesticides satisfying the recovery criteria in this procedure was slightly lower than when a defatting procedure was not applied (see Table 2). The scatter plot between recovery and RSD (Fig. 2) for individual pesticides indicated that a non-partitioning procedure resulted in better recovery and a more constant RSD than when a partitioning procedure was applied. Some of the pesticides with a higher $\log \mathrm{P}$ remained in the hexane layer during partitioning, resulting in low recovery. To verify the cleanup efficiency, scan chromatograms of control soybean samples were compared (Fig. S1). At $t_{\mathrm{R}}$ values of 5.0-10.0 $\mathrm{min}$ and $26.5-27.0 \mathrm{~min}$, more impurities were detected when the non-partitioning procedure was applied than when the partitioned procedure was applied, whereas larger peaks were observed when the partitioning procedure was applied at a $t_{\mathrm{R}}$ value of $31.0 \mathrm{~min}$ or greater. There were no significant differences in the cleanup between the procedures. From the results, the preparation procedures without $n$-hexane/acetonitrile partitioning (non-partitioning) were considered as the most appropriate soybean treatment methods for GC-MS/MS analysis.

\section{Matrix effect}

Matrix effect is a common phenomenon when analyzing pesticides via GC [21]. A scatter plot between the $t_{\mathrm{R}}$ and matrix effect (Fig. 3) indicated that the signals of most analytes were enhanced by co-eluent matrices throughout the $t_{\mathrm{R}}$. Many previous studies have reported signal enhancement by crop matrices in GC-MS or GC-MS/MS [22-24]. The average matrix effect for target pesticides was $5.5 \%$. The range of the matrix effect was classified into six groups with three categories (soft, medium, and strong effect) as shown in Fig. 4 [25, 26]. The most of the
Fig. 4 Distribution of matrix effects (\%) for 203 pesticides

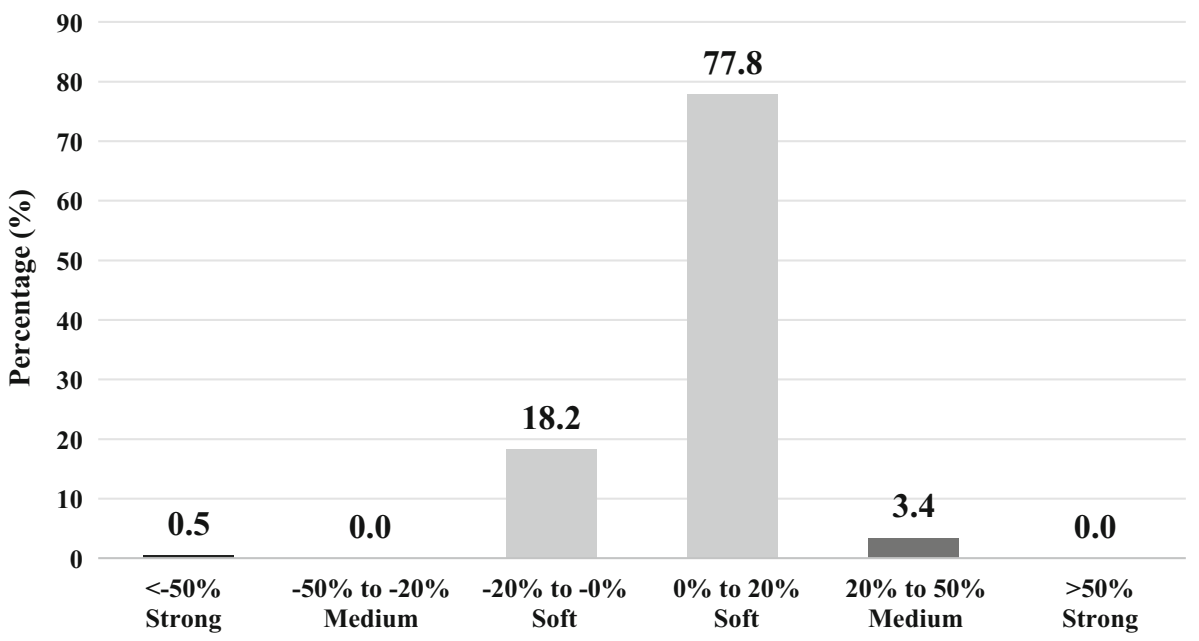

Matrix effect range 
pesticides $(96.0 \%)$ were included in the soft matrix effect range between -20 and $20 \%$. Pesticides within this effect range do not require matrix-matching because this effect is negligible [27]. However, matrix-matching is required for compounds in the medium (matrix effect between -50 to $-20 \%$ or 20 to $50 \%)$ and strong $(<-50 \%$ or $>50 \%)$ groups correct quantitation via GC-MS/MS [28]. The matrix effect values for the 203 pesticides are given in Table S3.

Acknowledgments This study was conducted with financial support from the Korea Ministry of Food and Drug Safety (16162MFDS020).

\section{References}

1. Ministry of Food and Drug Safety. Positive List System for pesticide residues in food. http://www.mfds.go.kr/. Accessed 1 June 2018

2. Iwasaki M, Sato I, Jin Y, Saito N, Tsuda S (2007) Problems of positive list system revealed by survey of pesticide residue in food. J Toxicol Sci 32:179-184

3. Grande-Martínez Á, Arrebola-Liébanas FJ, Martínez-Vidal JL, Hernández-Torres ME, Garrido-Frenich A (2016) Optimization and validation of a multiresidue pesticide method in rice and wheat flour by modified QuEChERS and GC-MS/MS. Food Anal Method 9:548-563

4. He Z, Wang Y, Wang L, Peng Y, Wang W, Liu X (2017) Determination of 255 pesticides in edible vegetable oils using QuEChERS method and gas chromatography tandem mass spectrometry. Anal Bioanal Chem 409:1017-1030

5. Shabeer TPA, Girame R, Utture S, Oulkar D, Banerjee K, Ajay D, Arimboor R, Menon KRK (2018) Optimization of multiresidue method for targeted screening and quantitation of 243 pesticide residues in cardamom (Elettaria cardamomum) by gas chromatography tandem mass spectrometry (GC-MS/MS) analysis. Chemosphere 193:447-453

6. Ministry of Food and Drug Safety. Korea Food Code [in Korean]. http://www.foodsafetykorea.go.kr. Accessed 16 May 2018

7. Żwir-Ferenc A, Biziuk M (2006) Solid phase extraction technique - trends, opportunities and applications. Pol J Environ Stud 15:677-690

8. Torres CM, Picó Y, Mañes J (1996) Determination of pesticide residues in fruit and vegetables. J Chromatogr A 754:301-331

9. Hartman GL, West ED, Herman TK (2011) Crops that feed the World 2. Soybean-worldwide production, use, and constraints caused by pathogens and pests. Food Sec 3:5-17

10. USDA. USDA Food Composition Databases. https://ndb.nal. usda.gov/ndb/search/list. Accessed 15 May 2018

11. Ministry of Food and Drug Safety (2017) Pesticide MRLs in Food [in Korean]. http://www.mfds.go.kr/. Accessed 16 May 2018

12. European Commission (2018) EU Pesticides database (files updated on 09/03/2018). http://ec.europa.eu/food/plant/pesti cides/eu-pesticides-database/public/?event=download.MRL. Accessed 1 June 2018

13. De Bièvre P, Günzler H (eds) (2005) Validation in chemical measurement. Springer, New York
14. Lee J, Kim E, Shin Y, Lee J, Lee J, Maasfeld W, Kim J-H (2018) Validation protocol for whole-body dosimetry in an agricultural exposure study. Appl Biol Chem 61:125-130

15. Zhao L, Lee HK (2001) Application of static liquid-phase microextraction to the analysis of organochlorine pesticides in water. J Chromatogr A 919:381-388

16. European Commission (2017) Guidance document on analytical quality control and validation procedures for pesticide residues and analysis in food and feed (SANTE/11813/2017). https://ec. europa.eu/food/sites/food/files/plant/docs/pesticides_mrl_guide lines_wrkdoc_2017-11813.pdf. Accessed 16 May 2018

17. Gunther FA, Blinn RC, Carman GE (1962) Insecticide residuesresidue of sevin on and in lemons and oranges. J Agric Food Chem 10:222-223

18. Mutavdžić D, Babić S, Ašperger D, Horvat AJM, Kaštelan-Macan M (2006) Comparison of different solid-phase extraction materials for sample preparation in the analysis of veterinary drugs in water samples. JPC-J Planar Chromat-Mod TCL 19:454-462

19. Páleníková A, Martínez-Domínguez G, Arrebola FJ, RomeroGonzález R, Hrouzková S, Frenich AG (2015) Multifamily determination of pesticide residues in soya-based nutraceutical products by GC/MS-MS. Food Chem 173:796-807

20. Chamkasem N, Ollis LW, Harmon T, Lee S, Mercer G (2013) Analysis of 136 pesticides in avocado using a modified QuEChERS method with LC-MS/MS and GC-MS/MS. J Agric Food Chem 61:2315-2329

21. Hajšlová J, Zrostlíková J (2003) Matrix effects in (ultra)trace analysis of pesticide residues in food and biotic matrices. J Chromatogr A 1000:181-197

22. Lee J, Kim L, Shin Y, Lee J, Lee J, Kim E, Moon J-K, Kim J-H (2017) Rapid and simultaneous analysis of 360 pesticides in brown rice, spinach, orange, and potato using microbore GC-MS/ MS. J Agric Food Chem 65:3387-3395

23. Lehotay SJ, Son KA, Kwon H, Koesukwiwat U, Fu W, Mastovska K, Hoh E, Leepipatpiboon N (2010) Comparison of QuEChERS sample preparation methods for the analysis of pesticide residues in fruits and vegetables. J Chromatogr A 1217:2548-2560

24. Schenck FJ, Lehotay SJ (2000) Does further clean-up reduce the matrix enhancement effect in gas chromatographic analysis of pesticide residues in food? J Chromatogr A 868:51-61

25. Kmellár B, Fodor P, Pareja L, Ferrer C, Martínez-Uroz MA, Valverde A, Fernandez-Alba AR (2008) Validation and uncertainty study of a comprehensive list of 160 pesticide residues in multi-class vegetables by liquid chromatography-tandem mass spectrometry. J Chromatogr A 1215:37-50

26. Ferrer C, Martínez-Bueno MJ, Lozano A, Fernández-Alba AR (2011) Pesticide residue analysis of fruit juices by LC-MS/MS direct injection. One year pilot survey. Talanta 83:1552-1561

27. He Z, Wang L, Peng Y, Luo M, Wang W, Liu X (2015) Multiresidue analysis of over 200 pesticides in cereals using a QuEChERS and gas chromatography-tandem mass spectrometrybased method. Food Chem 169:372-380

28. Kmellár B, Abrankó L, Fodor P, Lehotay SJ (2010) Routine approach to qualitatively screening 300 pesticides and quantification of those frequently detected in fruit and vegetables using liquid chromatography tandem mass spectrometry (LC-MS/MS). Food Addit Contam A Chem Anal Control Expo Risk Assess 27:1415-1430 